\title{
Born-Open Data for E-Prime
}

\author{
Denis Cousineau \\ Université d'Ottawa
}

\begin{abstract}
Born-Open Data experiments are encouraged for better open science practices. To be adopted, Born-Open data practices must be easy to implement. Herein, I introduce a package for EPrime such that the data files are automatically saved on a GitHub repository. The BornOpenData package for E-Prime works seamlessly and performs the upload as soon as the experiment is finished so that there is no additional steps to perform beyond placing a package call within E-Prime. Because E-Prime files are not standard tab-separated files, I also provide an $\mathrm{R}$ function that retrieves the data directly from GitHub into a data frame ready to be analyzed. At this time, there are no standards as to what should constitute an adequate open-access data repository so I propose a few suggestions that any future Born-Open data system could follow for easier use by the research community.
\end{abstract}

\section{Introduction}

Born-Open data experimentation is an approach whereby data collected in research experiments are made available to the research community as soon as they are collected and as transparently as possible. First proposed by Rouder in 2016, it is part of open science initiatives meant to promote transparency and better practices (sns12). Other proposals for open science includes the Peer reviewer's openness initiative (Morey et al., 2016), preregistration (Open Science Colaboration, 2015), and open source movements, among others.

To be successful, a Born-Open Data implementation should be easy to use and transparent to the experimenters as much as possible. Otherwise, it may not be implemented for the unique reason that experimenters may not have enough time to learn subsidiary tasks such as making data openly accessible.

Herein, I propose a Born-Open Data system for E-Prime. E-Prime is among the most commonly used software for experimentation in psychology (Richard \& Charbonneau, 2009). The core of the present system is a package for EPrime. A package call has to be dropped anywhere in the experiment's time line using the icon PackageCall. Four parameters are then required to specify the repository. Once this is done, at the end of any session, E-Prime will upload automatically a copy of the participant's data on a publicly available repository, GitHub.com.

The present document also presents an $\mathrm{R}$ function that can be used to read the data from the GitHub server. Only two parameters are required to point to the desired repository. After this function is called, a data frame ready to be analyzed

This research was supported in part by le Conseil pour la recherche en sciences naturelles et en genie of Canada. is obtained.

Finally, from what I learned from the development of this Born-Open Data system, I examine what should be common to all open-data systems (be it for E-Prime or for other programming environments) so that the open data can be consulted efficiently and rapidly by anyone. But before I proceed, I present some background knowledge.

\section{Born-Open and open format; git and GitHub}

E-Prime is not an open source software and the data files created with it (files with extensions .edat 2 or .edat 3 depending on the version of E-Prime) are not in an open format (that is, they cannot be read without E-Prime). This is a major obstacle to Born-Open Data. Fortunately, E-Prime also creates a text file containing the data (for data recovery in case the experiment crashes). This file can be read with any text editor and - with minimal work - can be turned into a data frame ready to be analyzed (e. g., with the library rprime for R; Mahr, 2015, R Core team, 2018).

To favor data openness, the present system uploads both .edat and .txt files onto an online repository system. To do so - and following Rouder, 2016-, I chose the git protocol. Git (Chacon \& Straub, 2014) is a set of commands meant to store files in a repository. Every time a commit command is issued, a snapshot of the files is taken and incrementally stored in a database. Subsequently, it is possible to know when a file was added or modified; it is also possible to access prior versions of the files. GitHub, on the other hand, is a web site offering free repository spaces which can be controlled with git commands issued on your computer. 


\section{Using the BornOpenData package}

\section{Prerequisites}

To be able to upload data files to a GitHub repository, three prerequisite are needed. First, it is necessary to be a registered user of the GitHub web site. Once registered, you must also create a repository that will receive the data files. Registration and open-access repositories are free of charge. Second, you also need to install Git on your computer. I used git version 2.20 for Windows, available from gitforwindows.org. Git is open source and free of charge. Finally, the computer must be online when the experiment is running.

For example, you could create a user for your laboratory, whose user name could be My-Laboratory. Once settled, you can create from that user a repository called JoeExperiment1. Hereafter, My-Laboratory will be referred to as the "owner" of the repository because he is the creator and the only one who can grant access to users who wishes to add new material to this repository (access to read the repository is granted to anyone unless you payed to have a private repository). The owner can for example grant access to another GitHub user, Joe, the one in charge of programming and running the experiment.

\section{Turning the experiment into a Born-Open Data experi- ment.}

To have the experiment performing uploads to GitHub, Joe only has to modify the experiment using E-Studio to insert the BornOpenData package into the experiment and then make a package call using a PackageCall icon. The two steps are described next. Note that E-Prime recommends that the name of the PackageCall resemble the name of the function that will be called (see next), so you may call it Call2RunExperimentThenUpload. Figure 1 shows a screen capture where the package call has been inserted in the experiment and renamed.

To insert a package into an experiment, you must go to the menu Edit: Experiment, and get to the "Packages" tab. Use the "Add" button to select the package called BornOpenData.epkg2. Figure 2 shows the result. If E-Prime does not find the package, you must go to the menu Tools: Options and the tab "Packages" and add the folder containing the package file BornOpenData.epk2 to the Package File search folder. This is done in Figure 3. I recommend that the package be located in the same folder as your experiment.

To make a package call, drag and drop the PackageCall icon. It can be inserted anywhere in the experiment. Double-clicking on the icon, you get the Properties dia$\log$ window. You must select the "BornOpenData" package in the drop-down list of available packages. In E-Prime, a package is a collection of functions. The only necessary function in the package BornOpenData is called RunExperimentThenUpload (the other function,
RunTestsThenAbort, is briefly discussed in the appendix). A package also contains scripts that are executed when the experiment is launched and just before an experiment is ended (the upload of the data to GitHub is actually performed during that last stage).

To operate, this function requires four arguments:

1. The GitHub username who will be receiving the data files. It could be the owner. However, because the password will also be required, it is preferable that the owner of the repository grants access to specific users. These users will be able to upload data files but won't be capable of permanently erasing the whole repository (only the owner can do that).

2. The password of that user. Note that the password is not encrypted so that anyone who can read the experiment can see the password. Remove it before sharing the experiment or change the password once the experiment is completed.

3. The name of the repository, and finally,

4. The user name of the owner of the repository.

Alternatively, you can put attributes in the package call with the usual [attributeName] notation. Thus, you could use start-up information. However, if the password is a startup information, then this attribute will be stored in all the data files.

Figure 4 shows the result for Joe's experiment 1.

To check that the third and fourth parameters are correct, you should be able to navigate to the following URL: https://github.com/owner/repository. Within the above example, that would be https://github.com/MyLaboratory/JoeExperiment1.

The function sets the upload parameters, but upload per se is done after the experiment is finished. Note that the upload takes from 10 to 30 seconds, so make sure that no one closes the computer in the meanwhile. Your last event in the experiment could for example be a Text Display showing "Thank you for your participation. Please report to the experimenter now (do not shut down the computer)" or similar. The last display remains on screen while upload is being performed.

Also, once the experiment created with E-Studio is transformed into an E-Basic script (file .ebs2), the script alone is needed to run the experiment. Thus, the package does not need to be copied to experimental stations.

This package performs two tasks: (i) it uploads the data file onto the GitHub repository within a folder called "rawdata" (it will be created if it does not exist); (ii) it creates or updates a file called "subjectsLog.txt", also on the GitHub repository, in which a new line is added every time a new participant has completed the experiment. The line contains: Experiment name (i.e., the E-Prime .es2 file name), Subject 
Figure 1. Screen capture of E-Studio showing a PackageCall object inserted in an experiment called AnExperiment.es2. The PackageCall has been renamed "Call2RunExperimentThenUpload"

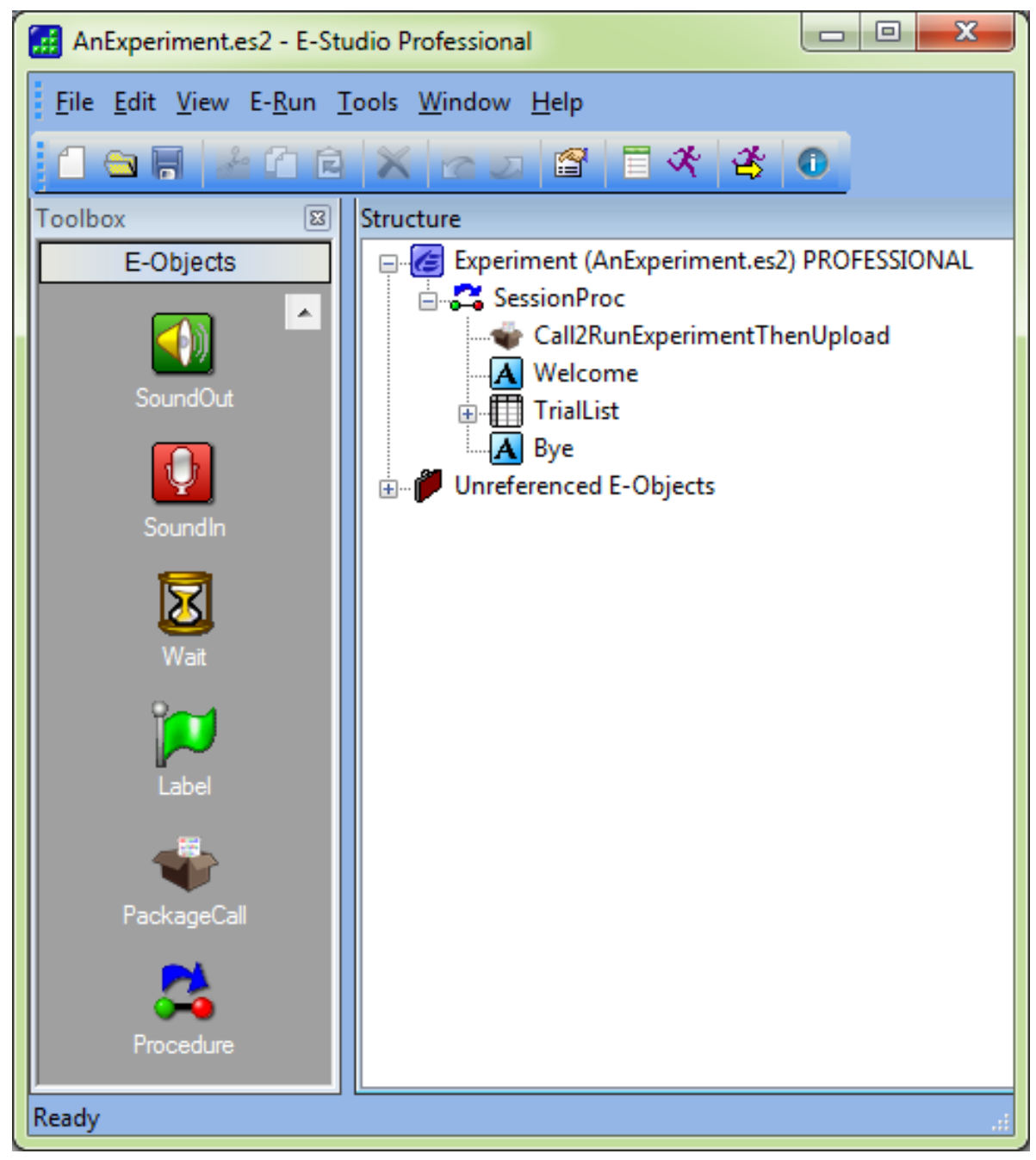

number, session number, date, time, computer name and status. This information is tab-separated so that it can be parsed by computer programs easily.

Finally, note that the package is uploading two data files. The data from the session are contained in a .edat2 file. However, as said earlier, the format of this file is not an openformat file so that it is not readable if you do not own EPrime. The package therefore also uploads a text file created by E-Prime. This file is a plain ASCII file easily read by anyone but is not in a convenient format (it is not in a tabular format with one line per trial). In the next section, I describe a tool to fetch and restructure these files.

\section{Importing the data into $\mathbf{R}$}

The GitHub repository will contain all the data from the experiment, one file per participant and per session. The data are contained in both an .edat 2 files and a .txt file created by E-Prime. Because this text file is not conveniently formatted, I propose an $\mathrm{R}$ function that can upload the data from the GitHub and turn them into a data frame convenient for analyses. It expands the rprime library (which does not generate a single table from the multiple data layers Mahr, 2015). Those possessing an E-Prime license can ignore this function, download the edat files and use E-DataAid as usual.

To have access to the function, you can install the following library (which contains a single function):

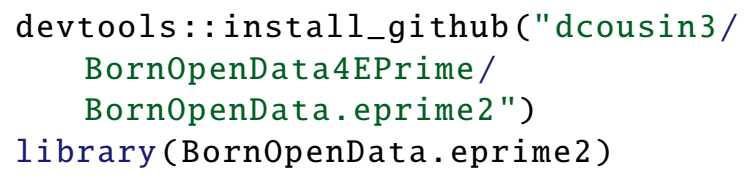

The first instruction, which assumes that the library de- 
Figure 2. The Edit: Experiment dialog with the "Packages" tab shown. The package has been inserted in the current experiment (the checkbox is checked).

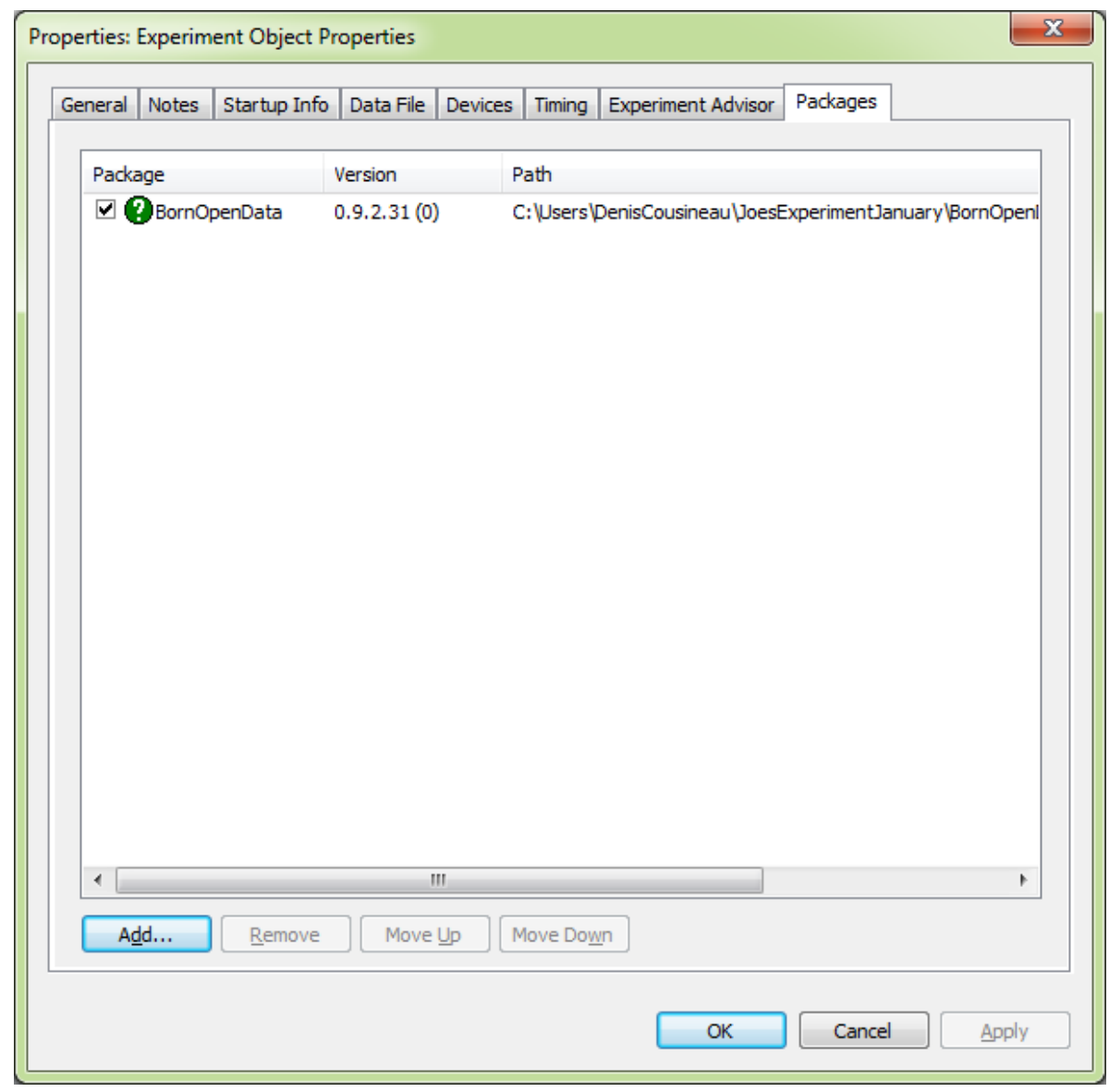

vtools is present on your computer, can be done only once prior to the first use.

After this is done, you read the data with

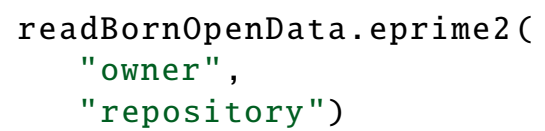

The data frame will be nearly identical to the text file exported by E-DataAid. For instance, with the above example, the command would be

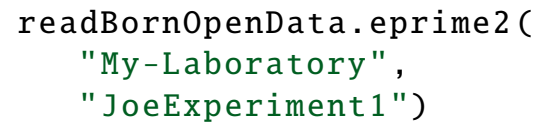

Note that $\mathrm{R}$ is case sensitive. The result is a data frame with all the subjects and all the sessions. The function fetches the data in the "rawdata" folder after consulting "subjectsLog.txt" to determine the files to be retrieved.

In the package call, four parameters were required to push the data onto GitHub. However, because the data are accessible to everyone, no user name and no password are required to read the data. This explains why the $\mathrm{R}$ function requires only two parameters.

Note that the current version of the function works for experiments with two layers only (trials within experiment) which is the typical experiment. A new version is underway for any number of layers.

\section{Requirements for a true Born-Open-Data system}

While working on this project, I made a certain number of observations as to what would be a well-structured repository, easily parsed by external humans and external computers. In my opinion, a Born-Open Data experiment must store data in a publicly available repository in such a way that any researcher can access those data at anytime from anywhere and be ready for analyses. Consequently, a Born-Open Data system should

1. Provide an easy-to-use add-on to an experiment that stores the experimental data

(a) in an open-access repository; 
Figure 3. The Tools: Options... dialog with the "Packages" tab shown. The location on the local computer of the experiment is added to the PackageFile search folders.

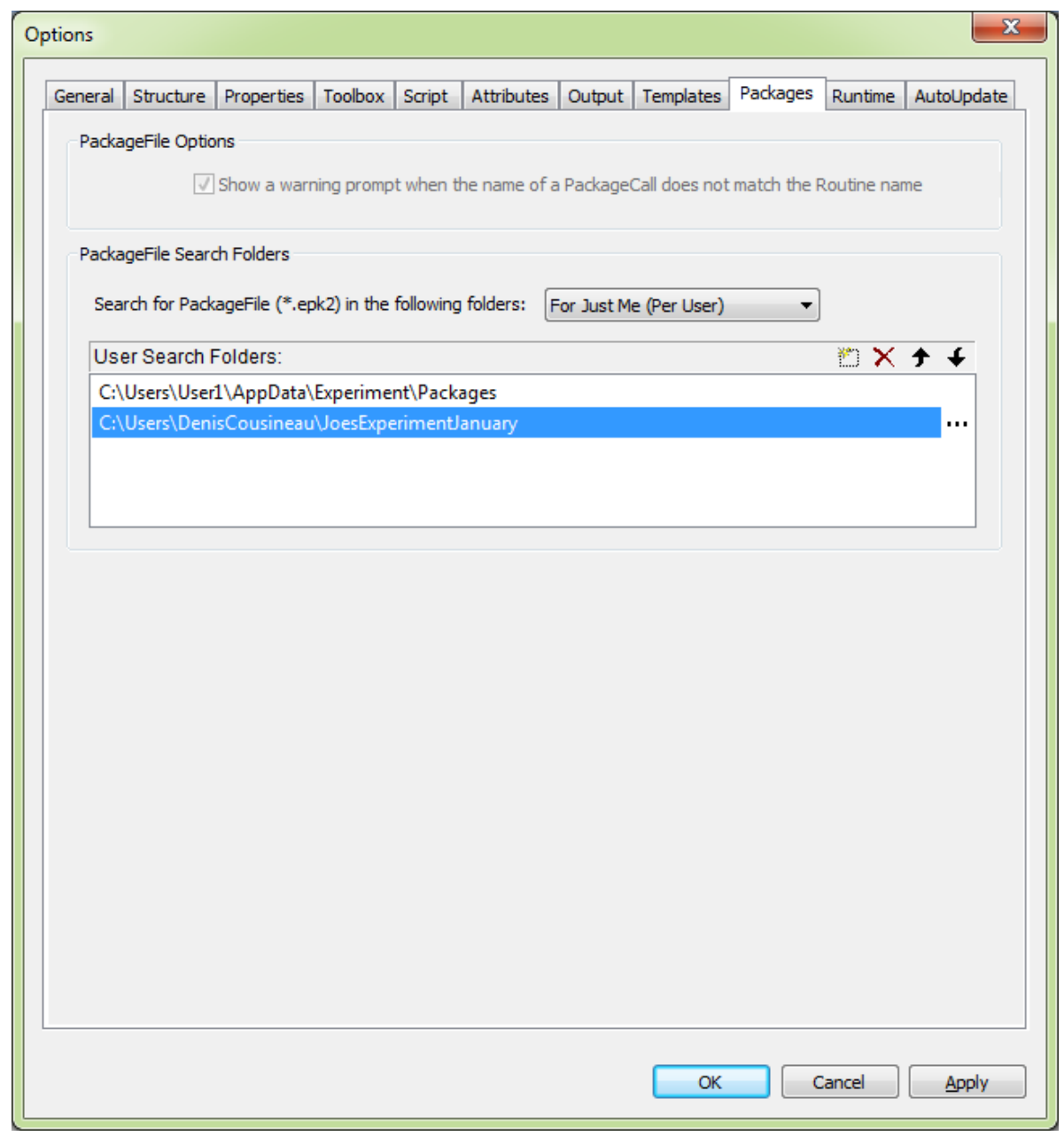

(b) in a predictable way;

(c) in a human and computer readable format;

(d) and preserves a human/computer readable log of the activity;

2. Provide a procedure that

(a) gets the data from the repository onto a statisti$\mathrm{cal} /$ programming environment

(b) in a convenient, ready-to-analyze format.

The present system for E-Prime complies with the above by (a) using GitHub as a repository. There exists other systems and in a future version, it would be convenient that the user can specify which repository web site to use. (b) the data files are stored, one per subject and per session in a subfolder called "rawdata", both as a .edat2 file and as a .txt file so that (c) the second file is human readable. (d) The file "subjectsLog.txt" contains a log of the uploaded files. Because these text files are not conveniently formatted, an R function to (e) import and (f) restructure the data is also provided.

A convenient repository structure therefore could resemble the one shown in Figure 5. In addition to SubjectsLog.txt, a file called VariablesLog.txt in which all the variables are described could be added to the repository by the researcher.

\section{Conclusion}

The BornOpenData for E-Prime is publicly available on https://GitHub.com/dcousin3/BornOpenData4EPrime. It contains two folders. The first one contains the E-Prime package for E-Prime version 2 (a file BornOpenData.epk2). The package can be opened by anyone possessing an EPrime license. I also put there a PDF document showing the codes in each section of the package for the benefits of those 
Figure 4. The properties of the Cal12RunExperimentThenUpload package call inserted in the experiment (Figure 1). The parameters indicate that the GitHub user "Joe" will perform uploads into the "JoeExperiment1" repository created by the GitHub user "My-Laboratory".

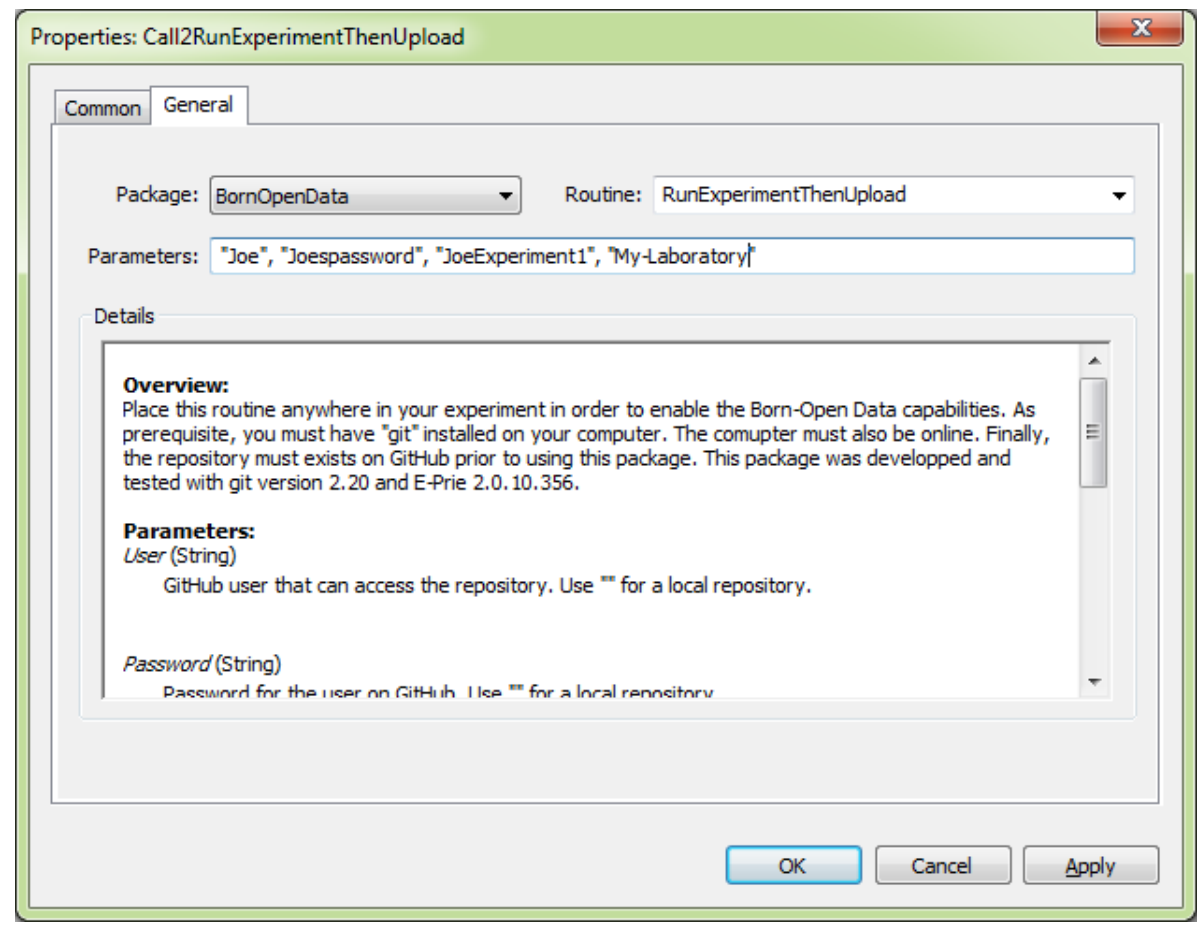

who do not have an E-Prime license.

The present Born Open Data system is slightly different from the system sketched in Rouder (2016). First, upload of data files is performed immediately and initiated by the experiment itself. Rouder suggested to perform uploads by night with a scheduled task not related to the experiment. Second, it is here suggested to have a repository for each experiment whereas Rouder suggested a single repository with multiple folders, one per experiment. The present approach allows managing accesses differently for each experiment. However, the owner must create a new repository manually for each experiment and manually grant access to selected users.

Born-Open Data systems for psychopy (Pierce, 2009) and its relative, OpenSesame (Mathôt, Schreij, \& Theeuwes, 2012), and other programming environments (Stahl, 2006) would be welcome so that integrating Born-Open data capabilities in an experiment becomes a simple formality.

It is sad that E-Prime data files are not in a publicly available format. I hope that PST will adapt to the new forces driving open science.

The present package for E-Prime has one limitation: Only GitHub (or a local folder on the computer) can be used as host for the repository. A future version will allow users to choose other git-compatible web servers.
The present package offers one interesting advantage with regards to multisite experimentations. Indeed, all the data collected from various sites are all uploaded to a single repository. Thus, there is no need to send local data files to an experiment coordinator. The subjectsLog.txt file contains the computer name which uploaded the data. To keep track of the computers, a new file, computersLog.txt, could be added to the repository which indicates for each computer identifiers (recorded in the SubjectsLog.txt file) their locations and their specifics.

\section{References}

Chacon, S., \& Straub, B. (2014). Pro git (2nd edition). Washington: APress.

Mahr, T. (2015). Rprime: Functions for working with 'eprime' text files (Version R package version 0.1.0). Retrieved from https://CRAN.R-project.org/package= rprime

Mathôt, S., Schreij, D., \& Theeuwes, J. (2012). Opensesame: An open-source, graphical experiment builder for the social sciences. Behavior Research Methods, 44, 314324. doi:10.3758/s13428-011-0168-7

Morey, R. D., Chambers, C. D., Etchells, P. J., Harris, C. R., Hoekstra, R., Lakens, D., . . Zwann, R. A. (2016). The peer reviewers' openness initiative: Incentivizing open 
Figure 5. A possible directory structure of the JoeExperiment1 repository showing folders and files that are likely to be found in an Open Data repository.

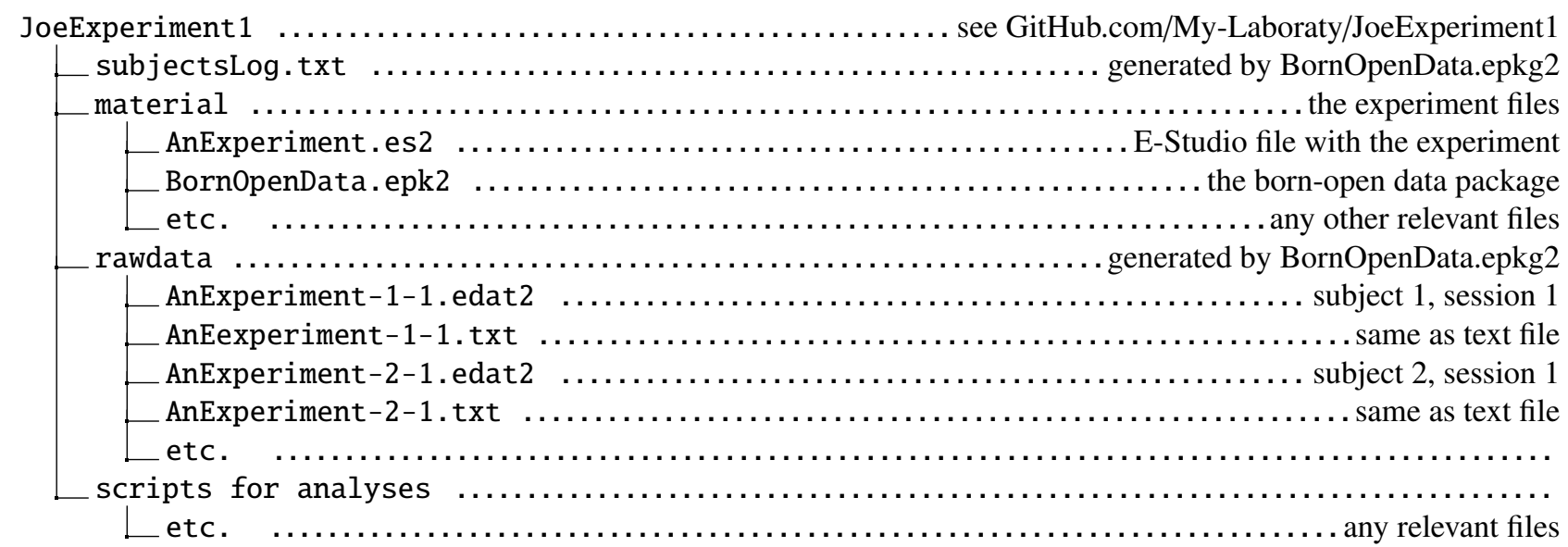

research practices through peer review. Royal Society Open Science, 3, 150547-1-150547-7. doi:10.1098/ rsos. 150547

Open Science Colaboration. (2015). Estimating the reproducibility of psychological science. Science, 349 , aac4716-1-aac4716-8. doi:10.1126/science.aac4716

Pierce, J. W. (2009). Generating stimuli for neuroscience using psychopy. Frontiers in NeuroInformatics, 2, 1-8. doi:10.3389/NEURO.11010.2008

R Core Team. (2018). R: A language and environment for statistical computing (technical report series no. 900051-07-0, URL Vienna, Austria: R Foundation for Statistical Computing. Retrieved from http://www.Rproject.org/)

Richard, L., \& Charbonneau, D. (2009). An introduction to eprime. Tutorials in Quantitative Methods for Psychology, 5, 68-76. doi:10.20982/tqmp.05.2.p068

Rouder, J. N. (2016). The what, why and how of bornopen data. Behavior Research Methods, 48, 10621069. doi:10.3758/s13428-015-0630-z

Stahl, C. (2006). Software for generating psychological experiments. Experimental Psychology, 53, 218-232.

\section{Appendix: Testing the installation}

The package BornOpenData for E-Prime contains a second function, RunTestsThenAbort. The purpose of this function is to perform a few basic tests then interrupt the experiment. Ideally, place this package call first in the experiment because the experiment will be interrupted right after the package call. This function requires the same four param- eters as the RunExperimentThenUpload function. Because of the abortion, error messages letting you know that the data could not be saved is normal.

The tests performed are, in that order:

1. Can E-Prime send commands to the terminal in the background (i.e., to cmd.exe)

2. Is git installed?

3. Is internet accessible?

4. Can the user access the owner's repository? This step mangles three verifications: Does the user exist on GitHub? Does the owner exist on GitHub? and does the repository exist under the owner's name?

5. and

6. Can the user perform local git operations (git add and git commit)? There would be problems here if the local folder is read only, for example. Finally,

7. Can the user push changes to the GitHub repository? Essentially, this test checks that the password provided is correct and that this user has been granted the right to modify the repository.

The test results should be visible in E-Studio in the Debug output tab. Also, if successful, a small file should appear in the repository, called "testBornOpenData.txt" in which the E-Studio experiment name, the computer name, the time and date should be indicated. This file can be erased. 